\title{
ALOCAÇÃo DE CUSTOS PELO MÉTOdO RECÍPROCO
}

\author{
Autor: Luiz João Corrar \\ Doutor em Controladoria e Contabilidade pela FEA/USP \\ Professor do Departamento de Contabilidade e Atuária
}

\section{Introdução}

O objetivo deste trabalho é mostrar o que é o método recíproco de alocação de custos, suas vantagens e como ele pode ser operacionalizado com a utilização de microcomputadores.

\section{A Contabilidade de Custos}

Os custos são apurados por centro de custo, que é apenas uma unidade contábil para a qual se acumulam custos (Martins, 1987).

Existem dois tipos de centros de custos:

- Centros de Custos de Produção, através dos quais passam os produtos. São unidades responsáveis pela produção dos bens normalmente destinados à venda. Podem ser denominados, também, Centros de Custos Fim;

- Centros de Custos de Serviços, que incorrem em custos mas não trabalham diretamente o produto. São responsáveis pela prestação de serviços aos demais centros de custos. Como tal, eles providenciam assistência aos centros de Custos Produtivos e aos outros centros de Custos de Serviços. Por exemplo: Manutenção, Almoxarifado, Contabilidade, Compras e outros. Esses centros de custos não participam, diretamente, nos processos de fabricação e seus custos são alocados aos centros de custos produtivos através de métodos, desde os mais simples aos mais sofisticados.

Essa alocação de custos é realizada quando o objetivo é estabelecer o custo dos produtos. Para esse efeito, numa primeira fase, os custos são alocados aos centros de custos de serviços entre si e também aos de produção. Numa segunda fase, todos os custos dos centros de serviços são eliminados pela distribuição aos centros produtivos. Finalmente, os custos acumulados nos centros produtivos são alocados aos produtos.

Uma preocupação que envolve os responsáveis pela atribuição dos custos aos produtos finais está na alocação dos custos diretos dos departamentos de serviços entre si e aos departamentos produtivos.

Estabelecem-se critérios de rateio dos custos diretos desses departamentos de serviços entre si e aos departamentos produtivos, que envolverão uma subjetividade possível de discussão. Entretanto, sem a adoção desses critérios de rateio torna-se impossível a apropriação dos mesmos ao produto final, salvo se esses custos diretos de departamentos de serviços existem unicamente para um processo produtivo, sendo a ele atribuídos totalmente, sem necessidade de rateio.

Verifica-se que, com grande freqüência,. determinados departamentos de serviços utilizam e fornecem simultaneamente serviços, bem como para outros departamentos de produção.

A interação entre os departamentos é que requer que a distribuição dos custos entre os departamentos seja feita de forma recíproca.

Caderno de Estudos nº12, São Paulo, FIPECAFI, Setembro/1995 
Antes do advento dos computadores, os contadores tendiam a ignorar esses relacionamentos recíprocos e simplesmente descarregavam todos os custos dos departamentos de serviços diretamente aos departamentos produtivos.

Outro método tradicional de rateio bastante utilizado é o de degraus.

Segundo este método deve-se escolher um departamento de serviço para transferir seus custos primários, conforme algum critério. Geralmente se escolhe o departamento que presta serviços a um maior número de outros departamentos.

Qualquer desses critérios não leva em conta os fluxos de serviços entre os departamentos.

O departamento escolhido para ser encerrado primeiro, é como se nenhum serviço recebesse dos demais, o que é falso, pois um departamento, além de prestar e receber serviços para outros e de outros, ainda pode prestar serviços a si próprio.

Atualmente existem programas de computadores desenvolvidos que facilitam a adoção desses conceitos.

Assim, foi possível incorporar os relacionamentos recíprocos no processo de alocação de custos, através da aplicação do cálculo matricial, cujas rotinas podem ser programadas em computadores.

Os motivos fundamentais para o melhor reconhecimento possível das interações entre os departamentos de serviços, e entre os departamentos de serviço e os de produção são.

- o método de distribuição de custos utilizado tem impacto no cálculo do custo dos departamentos de produção e, portanto, no cálculo do custo unitário e na margem de lucratividade de cada produto;

- proporciona o custeio com maior ou menor precisão dos produtos estocados e vendidos,

- impacta os custos dos serviços gerados, afetando a decisão: comprar ou fabricar o serviço.

\section{O Modelo de Alocação de Custos}

Suponha uma empresa em que existam dois Departamentos de Serviços (S1 e S2) e dois Departamentos de Produção (PI e P2), onde o Departamento de Serviço S1 presta serviço aos demais Departamentos de Serviço e de Produção. Mas recebe serviços do Departamento de Serviços S2.

Os Departamentos de Produção só recebem serviços e portanto não distribuem custos.

O Custo Total (CT) de cada departamento é igual ao Custo Direto (CD), mais Custo Indireto (CI), ou seja:

$$
C T=C D+C l
$$

Assim,

$$
\mathrm{CT}-\mathrm{Cl}=\mathrm{CD}
$$

Façamos:

$$
\mathbf{C T}=\mathbf{x}
$$

$$
\mathrm{Cl}=\mathrm{A} \cdot \mathrm{X}
$$

Caderno de Estudos nº12, São Paulo, FIPECAFI, Setembro/1995 
(onde "A" corresponde aos percentuais de alocação de custos entre os departamentos, sendo estes percentuais obtidos em função das bases de rateio estabelecidas)

$$
C D=b
$$

Assim,

$$
\begin{aligned}
& X-A X=b \\
& X(1-A)=b \\
& X \quad \frac{\equiv b}{1-A}
\end{aligned}
$$

Assim, tendo os Custos Diretos de cada departamento e os percentuais de distribuição de custos, obteríamos os Custos Totais de cada um deles. Entretanto,. devido ao fato de, geralmente, estar envolvido grande número de centros de custos nesse processo,. é necessário utilizar métodos que facilitem a operacionalização dos cálculos. Dai a utilização da álgebra matricial.

\section{A Álgebra Matricial}

Vamos tecer alguns breves comentários para poder entender como opera a álgebra matricial nesse caso.

Um sistema de equações lineares pode ser representado por notação algébrica ou por notação matricial (Shamblín et al, 1979).

Considere. por exemplo, o sistema de equações a seguir.

$$
\begin{array}{llll}
\mathbf{a}_{11} X_{1}+ & +\mathbf{a}_{12} X 2 & +\mathbf{a}_{13} X 3 & =b_{1} \\
\mathbf{a}_{21} X 1 & +\mathbf{a}_{22} X 2 & +\mathbf{a}_{23} X_{3}=b_{2} \\
\mathbf{a}_{31} X 1 & +\mathbf{a}_{32} X 2 & +\mathbf{a}_{33} X_{3}=b_{3}
\end{array}
$$

Esse sistema de equações pode ser representado por uma matriz e dois vetores coluna como segue:

\begin{tabular}{|lll|l|l|l|l|}
\hline $\mathbf{a}_{11}$ & $\mathbf{a}_{12}$ & $\mathbf{a}_{13}=$ & $\mathrm{X}_{1}$ & & $=\mathrm{b}_{1}$ \\
$\mathbf{a}_{21}$ & $\mathbf{a}_{22}$ & $\mathbf{a}_{23}=$ & $\mathbf{0}$ & $\mathrm{X}_{2}$ & & $=\mathrm{b}_{2}$ \\
$\mathbf{a}_{31}$ & $\mathbf{a}_{32}$ & $\mathbf{a}_{33}=$ & $\mathrm{b}_{3}$ \\
& & & $\mathrm{X}_{3}$ & & \\
\hline
\end{tabular}

Assim, temos na ordem em que aparecem:

- uma matriz de três linhas e três colunas representando os coeficientes das variáveis, 
- um vetor coluna de três linhas representando as três variáveis, ou incógnitas:

- um vetor coluna de três linhas representando as constantes.

Para verificar que a expressão acima é equivalente a anterior basta efetuar a multiplicação indicada, ou seja, da matriz pelo primeiro vetor coluna. Assim, temos:

\begin{tabular}{|lllll|l|}
\hline $\mathbf{a}_{11} X_{1}+\mathbf{a}_{12} X 2$ & + & $\mathbf{a}_{13} X 3$ \\
$\mathbf{a}_{21} X 1+\mathbf{a}_{22} X 2$ & + & $\mathbf{a}_{23} X_{3}$ \\
$\mathbf{a}_{31} X 1$ & $+\mathbf{a}_{32} X 2$ & $+\mathbf{a}_{33} X_{3}$ & & $=b_{3}$ \\
& & & & & $=b_{3}$ \\
& &
\end{tabular}

De maneira geral, equações lineares são expressas em forma matricial da seguinte maneira:

\begin{tabular}{|l|l|c|c|c|}
\hline Matriz dos coeficientes & $\bullet$ & $\begin{array}{c}\text { Vetor } \\
\text { Das } \\
\text { Variáveis }\end{array}$ & $=$ & $\begin{array}{c}\text { Vetor } \\
\text { Das } \\
\text { Constantes }\end{array}$ \\
\hline
\end{tabular}

As referidas equações podem ser escritas da seguinte forma resumida:

\section{A . $X=B$}

Onde:
A, representa a matriz dos coeficientes,
$\mathbf{X}$, representa o vetor coluna das variáveis,
B, representa o vetor coluna das constantes.

Se a álgebra matricial admitisse a operação de divisão nos mesmos moldes como é utilizada pela álgebra, seria bastante simples obter a solução para a expressão acima. Como não é possível a divisão de forma direta em álgebra matricial, é necessária a existência de um processo que permita obter resultados semelhantes. Assim, se tivéssemos a expressão:

$$
A B=C
$$

e multiplicássemos ambos os membros dessa expressão pelos termos 1/A (inverso de A) 


$$
\frac{1}{A} \times A B=\frac{1}{A} \quad x C
$$

obteríamos:

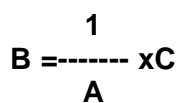

Dessa forma, com a operação de multiplicação, possível em álgebra matricial, e com utilização de um termo matricial que possua propriedades semelhantes ao inverso, torna-se viável a solução da questão. Em álgebra matricial existe esse termo denominado de inversa, que é notado por um expoente negativo.

Por exemplo:

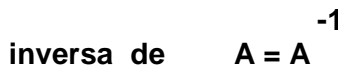

A inversa tem a seguinte propriedade:

$$
\stackrel{-1}{A} \cdot A=\stackrel{-1}{A} \cdot A=1
$$

Ou seja, a inversa de A multiplicada por A resulta na matriz-identidade representada por 1. Uma matriz identidade é uma matriz quadrada (aquela cujo número de linhas é igual ao número de colunas), que possui o número 1 na diagonal principal e zero no restante. Exemplo:

\begin{tabular}{|l|}
\hline 100 \\
010 \\
001 \\
\hline
\end{tabular}

Deve-se ressaltar que na multiplicação matricial a matriz-identidade tem propriedades semelhantes ao número 1 da multiplicação regular. Assim, qualquer matriz multiplicada por uma matriz-identidade tem, como seu produto, a matriz original.

Com base no que foi explicado, para resolver a expressão representativa do caso em estudo, ou seja:

$$
\text { A. } \mathbf{X}=\mathbf{B}
$$

bastaria multiplicar pela inversa ambos os membros da expressão, ou seja: 


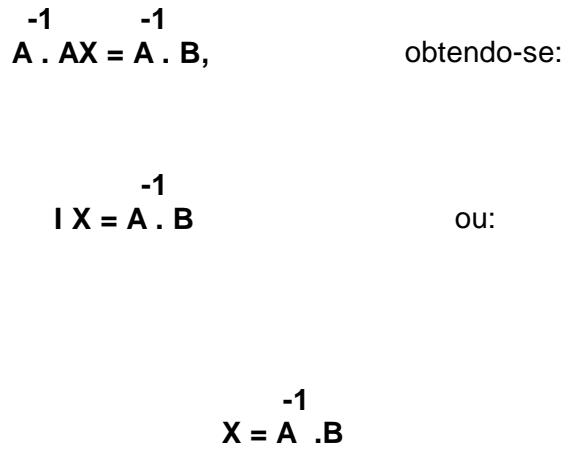

Em resumo, para solucionar a nossa questão, que é a de encontrar o valor das variáveis, bastaria determinar o valor da matriz-inversa dos coeficientes $(A)$ e multiplicar esse resultado pela matriz das constantes (B).

Entretanto, resta ainda um problema adicional, qual seja o de como determinar a matriz-inversa. Hoje em dia, porém, com os recursos computacionais existentes, o cálculo da matriz-inversa e mesmo multiplicação de matrizes, passou a não representar qualquer empecilho. Basta que seja possível dispor de um microcomputador e um programa que disponha de uma simples planilha eletrônica, como por exemplo: LOTUS 123, QUATRO PRO, EXCEL etc. Uma vez entendidos os conceitos emitidos neste tópico, a parte operacional é bastante simples e será aplicada, a seguir, através de um exemplo.

\section{Aplicação de Matrizes}

Suponha:

- dois Departamentos de Produção P1 e P2

- dois Departamentos de Serviço: S1 e S2 Custos Diretos dos Departamentos antes da alocação:

\section{U\$\$}

$$
\begin{aligned}
& \text { S1 }=9.000 \\
& \text { S2 }=6.000 \\
& P 1=20.000 \\
& P 2=16.000
\end{aligned}
$$

Os percentuais de distribuição de custos entre os centros de custos obtidos através de estudos técnicos (utilização efetiva dos serviços ou potencial de utilização) são:

Caderno de Estudos nº12, São Paulo, FIPECAFI, Setembro/1995 


$\begin{array}{crrrr}\text { De/Para } & \text { S1 } & \text { S2 } & \text { P1 } & \text { P2 } \\ \text { S1 } & 0,0 & 0,2 & 0,4 & 0,4 \\ \text { S2 } & 0,1 & 0,0 & 0,4 & 0,5 \\ \text { P1 } & 0,0 & 0,0 & 0,0 & 0,0 \\ \text { P2 } & 0,0 & 0,0 & 0,0 & 0,0\end{array}$

Voltando ao item 'O Modelo de Alocação de Custos”, onde obtivemos a expressão:

$$
x=\frac{b}{=1}-A
$$

e, observando o que foi explicado no item "Álgebra Matricial', a expressão anterior fica da seguinte forma, em cálculo matricial:

$$
x=(1-A) \cdot b
$$

\begin{tabular}{|c|c|c|c|c|c|c|c|c|c|}
\hline $\begin{array}{l}X 1 \\
X 2 \\
X 3 \\
X 4\end{array}$ & $=$ & $\begin{array}{l}1000 \\
0100 \\
0010 \\
0001\end{array}$ & $(-)$ & $\begin{array}{r}0 \\
0,2 \\
0,4 \\
0,4\end{array}$ & $\begin{array}{r}0,1 \\
0 \\
0,4 \\
0,5\end{array}$ & $\begin{array}{l}0 \\
0 \\
0 \\
0\end{array}$ & $\begin{array}{l}0 \\
0 \\
0 \\
0\end{array}$ & $(\bullet)$ & $\begin{array}{r}9.000 \\
6.000 \\
20.000 \\
16.000\end{array}$ \\
\hline $\mathbf{X}=$ & & I & $(-)$ & & $\mathbf{A}$ & & & 1 & b \\
\hline
\end{tabular}

Atribuindo os respectivos valores para a expressão acima, temos:

Considerando as coordenadas da planilha eletrônica transcrita abaixo e digitando os dados da matriz (1 A), vem:

A

$\begin{array}{ll}1 & 1 \\ 2 & -0,2 \\ 3 & -0,4 \\ 4 & -0,4\end{array}$

B

$-0,1$

1

$-0,4$

$-0,5$
C D

$0 \quad 0$

$0 \quad 0$

$\begin{array}{ll}1 & 0 \\ 0 & 1\end{array}$

Em seguida acionam-se os comandos do Lotus 123:

I $\quad$ para chamar o menu principal;

DATA opção onde se encontra o comando para se trabalhar com matrizes;

MATRIX para selecionar o comando operador de matrizes; 
INVERT para escolher a operação de inversão de matrizes;

A1.04 <ENTER> $>\quad$ para determinar a faixa de dados de entrada; e

E1 para determinar o local de saída dos dados.

Assim, obtêm-se os seguintes resultados:

E $\quad F$

$\begin{array}{lllll}1 & 1,020408 & 0,102040 & 0 & 0 \\ 2 & 0,204081 & 1,020408 & 0 & 0 \\ 3 & 0,489795 & 0,448979 & 1 & 0 \\ 4 & 0,510204 & 0,551020 & 0 & 1\end{array}$

Esses resultados representam os valores da denominada matriz - inversa

\section{$-1$ \\ $(1-A)$}

I

$\begin{array}{lr}1 & 9.000 \\ 2 & 6.000 \\ 3 & 20.000 \\ 4 & 16.000\end{array}$

O passo seguinte é digitar os dados do vetor $\mathbf{b}$. Os valores que aparecem na coluna 1 representam o denominado vetor das constantes $\mathbf{b}$.

Agora basta efetuar uma operação de multiplicação de matrizes para resolver o problema. Os passos são:

para chamar o menu principal;

DATA

opção onde se encontra o comando para se trabalhar com matrizes;

MATRIX

para selecionar o comando operador de matrizes;

MULTIPLY

para escolher a operação de multiplicação de matrizes;

E1.H4

para determinar a faixa de dados de entrada referentes à primeira matriz a ser multiplicada; e

I1.14 para determinar a faixa referente à segunda matriz a ser multiplicada; e 
Assim, na coluna $\mathbf{J}$ aparecerão os seguintes resultados:

$J$

$\begin{array}{ll}1 & 9.795,918 \\ 2 & 7.959,183 \\ 3 & 27.102,04 \\ 4 & 23.897,95\end{array}$

K

$\mathrm{X} 1$

$\mathrm{X} 2$

$\mathrm{X} 3$

$\mathrm{X} 4$

Na coluna K estão identificadas as incógnitas do problema através das respectivas notações utilizadas. Os valores correspondentes a cada uma delas estão automaticamente determinados na coluna $\mathbf{J}$.

Os valores: \$ 9. 795,918 para X1; \$ $7.959,183$ para X2; \$27.102,04 para X3 e \$23.897,95 para X4, representam os custos diretos do próprio departamento mais os custos indiretos recebidos dos demais.

As variáveis X1 e X2 representam os Departamentos de Serviços S1 e S2. As variáveis X3 e X4 representam os Departamentos de Produção P1 e P2.

Portanto, os valores de X3 e X4, contém os custos diretos dos Departamentos de Produção mais os indiretos recebidos dos Departamentos de Serviços.

\section{Conclusão}

O exemplo apresentado é bastante simplificado. Na realidade, as empresas possuem inúmeros centros de custos. Entretanto, o caso discutido é suficiente para entender o mecanismo da alocação de custos pelo método recíproco. A vantagem da alocação recíproca, segundo o Professor ludícibus (1988), é que esta respeita o efetivo fluxo de serviços entre os departamentos, sem deixar algum de fora, e sem assumir hipóteses baseadas apenas no bom senso, mas que nem sempre são completas para os rateios. 
Alocação de Custos pelo Método Recíproco 10

\section{Bibliografia}

1. IUDÍ́CIBUS, Sérgio de. Análise de Custos. São Paulo: Atlas, 1988.

2. MARTINS, Eliseu. Contabilidade de Custos. São Paulo: Atlas, 1987.

3. SHAMBLIN, J.E. e STEVENS Jr. G.T. Pesquisa Operacional. São Paulo: Atlas, 1979. 